\title{
Association of ABO blood groups with von Willebrand factor, factor VIII and ADAMTS-13 in patients with lung cancer
}

\author{
XIA LIU ${ }^{1}$, XIAOGANG CHEN ${ }^{2}$, JIEZUAN YANG ${ }^{3}$ and RENYONG GUO ${ }^{2}$ \\ Departments of ${ }^{1}$ Intensive Care Unit and ${ }^{2}$ Laboratory Medicine; ${ }^{3}$ State Key Laboratory for Diagnosis and Treatment of \\ Infectious Disease, First Affiliated Hospital, College of Medicine, Zhejiang University, Hangzhou, Zhejiang 310003, P.R. China
}

Received August 25, 2015; Accepted January 31, 2017

DOI: $10.3892 / \mathrm{ol} .2017 .6619$

\begin{abstract}
Coagulative and fibrinolytic disorders appear to be associated with the development of lung cancer. The aim of the present study was to determine plasma levels of von Willebrand factor (VWF) and a disintegrin and metalloproteinase with a thrombospondin type 1 motif 13 (ADAMTS-13), and factor VIII (FVIII) activity, in association with $\mathrm{O}$ and non-O blood groups in patients with lung cancer. Plasma levels of VWF and ADAMTS-13, and FVIII activity were measured in 115 patients with lung cancer and 98 healthy subjects. Phenotyping of the ABO blood groups was also performed for the two groups. Significantly increased VWF levels and FVIII activity, as well as significantly decreased ADAMTS-13 levels, were observed in patients with distant metastasis as compared with those without distant metastasis and the healthy controls. Plasma VWF levels and FVIII activity were significantly increased in subjects with non-O type blood compared with those with type O blood in the two groups. However, a significant decrease in ADAMTS-13 levels was observed only in the control group among those with non-O type blood, compared with those with type $\mathrm{O}$ blood. The results of the present study indicate that increased VWF and decreased ADAMTS-13 levels facilitate the invasiveness and metastasis of lung cancer. Non-O blood groups constitute a risk factor for increased VWF and FVIII in plasma. Continued monitoring of VWF and ADAMTS-13 levels, and of FVIII activity in patients with lung cancer with distinct blood groups may help to minimize the incidence of thrombotic events and improve assessment of disease progression.
\end{abstract}

Correspondence to: Dr Renyong Guo, Department of Laboratory Medicine, First Affiliated Hospital, College of Medicine, Zhejiang University, 79 Qingchun Road, Hangzhou, Zhejiang 310003, P.R. China E-mail: lhgry@126.com

Key words: lung cancer, a disintegrin and metalloproteinase with a thrombospondin type 1 motif 13, von Willebrand factor, factor VIII, ABO blood groups

\section{Introduction}

Lung cancer, which is characterized by subclinical activation of blood coagulation and fibrinolysis from the early clinical stages of disease, is the most common cause of cancer-associated mortality worldwide (1). Significant increases in the blood concentrations of thrombin-antithrombin III complexes, fibrinogen and D-dimer have been reported in patients with lung cancer exhibiting extensive or limited disease. The extent of such activation of these markers has also been associated with tumor stage and prognosis $(2,3)$. However, the origin of the activation of the coagulative-fibrinolytic system in lung cancer remains incompletely understood.

Von Willebrand factor (vWF) is a multimeric glycoprotein produced by the vascular endothelium and megakaryocytes that is crucial for hemostasis, and functions as a carrier protein for factor VIII (FVIII) and as a bridge between the subendothelial matrix and platelets (4). The distinctive properties of the distinct molecular masses of VWF influence its ability to mediate platelet adhesion and aggregation (5). Unusually large multimers stored in and released from Weibel-Palade bodies in stimulated vascular endothelial cells are the hemostatically active form of VWF (6). A disintegrin and metalloproteinase with a thrombospondin type 1 motif13 (ADAMTS-13), formerly known as VWF cleavage protease, is a metalloprotease that specifically cleaves the multimeric VWF into smaller fragments between $\mathrm{Tyr}^{1605}$ and $\mathrm{Met}^{1606}$ within the VWF A2 domain (7). The cellular origin of the ADAMTS-13 antigen in plasma has been described in platelets, and in hepatic, renal and endothelial cells. Deficiency of ADAMTS-13 activity results in an increase in circulating large VWF multimers (8).

An association between the ABO blood group and blood coagulation was recognized several years previously. Non-O blood type has been associated with a significantly increased risk of venous and arterial thromboembolic disease, including deep vein thrombosis, pulmonary embolism, ischemic heart disease and peripheral vascular disease (9). The plasma levels of two coagulation glycoproteins, VWF and FVIII, have been demonstrated as the basis of this association between the ABO blood group and blood coagulation (10). Individuals with type O blood have significantly decreased plasma levels of VWF and FVIII, compared with those with non-O blood types (A, B and AB) (11). In total, $66 \%$ of the variations in VWF 
plasma levels are associated with mutations, and 30\% of these are associated with the effect of the ABO blood group (10).

Increased levels of VWF and FVIII and impaired activity of ADAMTS-13 have been demonstrated in patients with metastasizing and malignant tumors (12). The majority of studies have not focused on the levels of VWF, FVIII and ADAMTS-13 in patients with lung cancer; however, Martini et al (13) reported that VWF antigen levels are not substantially altered in patients with non-small cell lung cancer. However, the imbalance in the distribution of $\mathrm{O}$ and non-O blood types between patients and healthy controls in that study may have influenced the results. Therefore, the aim of the present study was to identify possible associations between ABO blood group types and plasma levels of VWF and ADAMTS-13, and FVIII activity in patients with lung cancer in order to improve the characterization of potential links between blood type and coagulative activation in lung cancer.

\section{Materials and methods}

Patients. The present cross-sectional study included 115 patients with histologically confirmed lung carcinoma who visited the First Affiliated Hospital of Zhejiang University School of Medicine (Hangzhou, China) between March 2013 and May 2014, and 98 age- and gender-matched healthy controls selected from individuals who underwent routine health examinations at the Health Center of the First Affiliated Hospital. A pathological diagnosis of lung cancer was derived from biopsy or cytological specimens collected at bronchoscopy or transthoracic needle aspiration, or from surgical specimens. Disease in all patients wasclassified according to the revised World Health Organization classification of lung tumors and staged according to the revised tumor-node-metastasis staging system for lung cancer $(14,15)$. Patient characteristics are presented in Table I.

All subjects enrolled in the present study had normal liver, renal and cardiac function. The exclusion criteria included a history of chronic hypertension, diabetes, renal, hepatic, cardiovascular and autoimmune diseases, anticoagulant or corticosteroid use, arterial or venous thrombosis, renal transplantation, human immunodeficiency virus infection and pregnancy. The present study was performed in accordance with the principles embodied in the Declaration of Helsinki and was approved by the Institutional Research Ethics Committee of the First Affiliated Hospital of Zhejiang University. Written informed consent was obtained from all participants.

Sample collection. Venous blood samples were collected from all participants into 3 tubes early in the morning following overnight fasting at the time of clinical diagnosis and prior to any treatment. A tube containing $0.109 \mathrm{M}$ trisodium citrate $(9: 1, \mathrm{v} / \mathrm{v})$ was used for measuring the plasma levels of D-dimer, fibrinogen, VWF and ADAMTS-13, as well as FVIII activity. Plasma was obtained by immediately centrifuging the blood in a refrigerated centrifuge at 1,500 $\mathrm{xg}$ for $15 \mathrm{~min}$. A second tube coated with EDTA ( $2.4 \mathrm{mg} / 2 \mathrm{ml}$ venous blood) was used for immediate classification of the ABO blood group, and for determination of the hemoglobin concentration and platelet count. Blood was also collected in a third tube without any anticoagulant and was allowed to clot for the isolation of serum following centrifugation at $1,500 \mathrm{x}$ g for $10 \mathrm{~min}$ at $4^{\circ} \mathrm{C}$. All plasma and serum samples were divided into $1 \mathrm{ml}$ aliquots and stored at $-80^{\circ} \mathrm{C}$ until evaluation.

Assays. Phenotyping of the ABO blood groups was performed using a Galileo blood group analyzer (Immucor, Inc., Norcross, GA, USA), with anti-A and anti-B blood grouping reagents and reference cells $\mathrm{A}_{1}, \mathrm{~A}_{2}, \mathrm{~B}$ and $\mathrm{O}$ (Immucor, Inc.). Serum levels of lactate dehydrogenase (LDH) were determined using a Hitachi 7600 automated biochemistry analyzer (Hitachi, Ltd., Tokyo, Japan) with an International Federation of Clinical Chemistry method (Roche Diagnostics $\mathrm{GmbH}$, Mannheim, Germany) according to the manufacturer's protocol (16). Serum levels of carcinoembryonic antigen (CEA) were measured using a chemiluminescent microparticle immunoassay with the ARCHITECT i2000SR system (Abbott Laboratories, Abbott Park, IL, USA). Plasma levels of fibrinogen (Clauss method) (17), D-dimer (immunoturbidimetric method) (18) and FVIII activity (coagulation method) (19) were measured using the Sysmex CS-5100 coagulation analyzer (Sysmex Corporation, Kobe, Japan), with the Dade ${ }^{\circledR}$ Thrombin reagent, INNOVANCE ${ }^{\circledR}$ D-Dimer and Coagulation Factor VIII Deficient Plasma (all from Siemens AG, Munich, Germany), respectively. Plasma levels of VWF and ADAMTS-13 were measured using the vWF ELISA kit and ADAMTS-13 ELISA kit (R\&D Systems, Inc., Minneapolis, MN, USA), according to the manufacturer's protocol.

Statistical analysis. All statistical analyses were performed using SPSS for Windows software (version 16.0 J; SPSS, Inc., Chicago, IL, USA). Shapiro-Wilk tests were used to determine whether variables were normally distributed. Continuous variables are expressed as the mean \pm standard deviation, or as the median and the 25-75th percentiles. Comparisons of results between two groups were made using the Student's t-test or Mann-Whitney U test, where appropriate. Non-parametric $\chi^{2}$ tests were used to compare categorical variables. $\mathrm{P}<0.05$ was considered to indicate a statistically significant difference.

\section{Results}

Patient characteristics. Table I summarizes the clinical characteristics of the 213 subjects enrolled in the present study. No significant differences in age, gender, systolic blood pressure (SBP), diastolic blood pressure (DBP) or smoking status between patients with lung cancer and healthy controls were identified. However, body mass index (BMI) was significantly decreased in patients with lung cancer $(\mathrm{P}=0.024)$. Baseline histopathological characteristics and lung cancer stages are also presented in Table I.

$A B O$ distribution. ABO blood group distribution in patients demonstrated that 29 (25.2\%) were type O, 41 (35.7\%) were type A, 30 (26.1\%) were type B and $15(13.0 \%)$ were type AB. In the control group, $34(34.7 \%)$ subjects were type O, 24 (24.5\%) were type A, $33(33.7 \%)$ were type B and 7 (7.1\%) were type AB (Table I). Subjects with type A, B and AB blood were pooled into the non-O group. No significant difference 
Table I. Baseline characteristics of the study population.

\begin{tabular}{|c|c|c|c|}
\hline Variable & Patients with lung cancer $(n=115)$ & Healthy controls $(n=98)$ & P-value \\
\hline Age, years & $57.1 \pm 8.5$ & $55.1 \pm 7.4$ & 0.101 \\
\hline Male/female, $\mathrm{n}$ & $76 / 39$ & $58 / 40$ & 0.299 \\
\hline BMI, $\mathrm{kg} / \mathrm{m}^{2}$ & $22.2 \pm 2.7$ & $23.1 \pm 2.6$ & $0.024^{\mathrm{a}}$ \\
\hline $\mathrm{SBP}, \mathrm{mmHg}$ & $128.0 \pm 9.0$ & $127.2 \pm 8.9$ & 0.645 \\
\hline $\mathrm{DBP}, \mathrm{mmHg}$ & $80.2 \pm 6.7$ & $78.5 \pm 7.5$ & 0.578 \\
\hline Smoker/non-smoker, n & $32 / 83$ & $20 / 78$ & 0.216 \\
\hline Histology & & $\mathrm{N} / \mathrm{A}$ & \\
\hline NSCLC, n (\%) & $89(77.4)$ & & \\
\hline Squamous cell carcinoma, n (\%) & $29(25.2)$ & & \\
\hline Adenocarcinoma, n (\%) & $51(44.3)$ & & \\
\hline Others (unclassified), n (\%) & $9(7.8)$ & & \\
\hline SCLC, n (\%) & $26(22.6)$ & & \\
\hline Stage of NSCLC disease & & $\mathrm{N} / \mathrm{A}$ & \\
\hline Local (stages I + II), n (\%) & $12(10.4)$ & & \\
\hline Locally advanced (stage III), n (\%) & $31(27.0)$ & & \\
\hline Metastatic (stage IV), n (\%) & $46(40.0)$ & & \\
\hline \multicolumn{4}{|l|}{ SCLC } \\
\hline Limited, n (\%) & $17(14.8)$ & & \\
\hline Extensive, n (\%) & $9(7.8)$ & & \\
\hline \multicolumn{4}{|l|}{ Blood group } \\
\hline $\mathrm{O}, \mathrm{n}(\%)$ & $29(25.2)$ & $34(34.7)$ & 0.131 \\
\hline Non-O, n (\%) & $86(74.8)$ & $64(65.3)$ & 0.131 \\
\hline $\mathrm{A}, \mathrm{n}(\%)$ & $41(35.7)$ & $24(24.5)$ & 0.078 \\
\hline $\mathrm{B}, \mathrm{n}(\%)$ & $30(26.1)$ & $33(33.7)$ & 0.227 \\
\hline $\mathrm{AB}, \mathrm{n}(\%)$ & $15(13.0)$ & $7(7.1)$ & 0.158 \\
\hline
\end{tabular}

${ }^{a} \mathrm{P}<0.05$. Values are presented as the mean \pm standard deviation. Frequencies $(\%)$ were evaluated using a $\chi^{2}$ test. BMI, body mass index; DBP, diastolic blood pressure; SBP, systolic blood pressure; N/A, not applicable; NSCLC, non-small cell lung cancer; SCLC, small cell lung cancer.

in the frequency of blood type between the two groups was identified (Table I).

Hemostatic parameters. Hemostatic data are summarized in Table II. The platelet count and serum levels of hemoglobin, LDH, CEA, D-dimer, fibrinogen and VWF, and FVIII activity were significantly increased in all patients with lung cancer, compared with in healthy controls $(\mathrm{P}<0.05)$, whereas a significant decrease in ADAMTS-13 levels was observed in all patients with lung cancer, compared with the healthy controls $(\mathrm{P}=0.011)$. Similarly, regardless of the presence of distant metastasis, patients with lung cancer also exhibited increased plasma levels of hemoglobin, LDH, CEA, D-dimer, fibrinogen and VWF, and FVIII activity when compared with healthy controls $(\mathrm{P}<0.05)$. However, a significant increase in platelet count and a significant decrease in ADAMTS-13 levels were only observed in patients with lung cancer exhibiting distant metastasis, as compared with healthy controls $(\mathrm{P}<0.05)$. Levels of hemostatic parameters were further compared between patients with and without distant metastasis, which demonstrated significant increases in plasma levels of LDH, CEA,
D-dimer and VWF, and in FVIII activity, and a significant decrease in plasma levels of ADAMTS-13 (Table II).

Correlation between hemostatic parameters and $A B O$ blood groups. All subjects in the two groups were categorized as either type $\mathrm{O}$ or non-O blood groups to compare the clinical characteristics and levels of hemostatic parameters. As presented in Table III, no significant differences in age, gender, BMI, SBP, DBP and smoking status between individuals in the type $\mathrm{O}$ or non-O blood groups among patients with lung cancer and the healthy controls were identified. Similarly, no significant difference in the distribution of patients with distant metastasis between the type $\mathrm{O}$ and non-O blood groups was identified. Regarding hemostatic parameters, patients with lung cancer from the non-O blood group demonstrated a significant increase in VWF plasma levels $(\mathrm{P}=0.022)$ and FVIII activity $(\mathrm{P}=0.014)$, compared with those in the type $\mathrm{O}$ blood group. A significant increase was identified in plasma levels of fibrinogen ( $\mathrm{P}=0.004$; Table III) and VWF ( $\mathrm{P}=0.006$; Fig. 1A), and FVIII activity $(\mathrm{P}=0.003$; Fig. $1 \mathrm{~B})$ in the control group among those with non-O type blood, compared with those with 


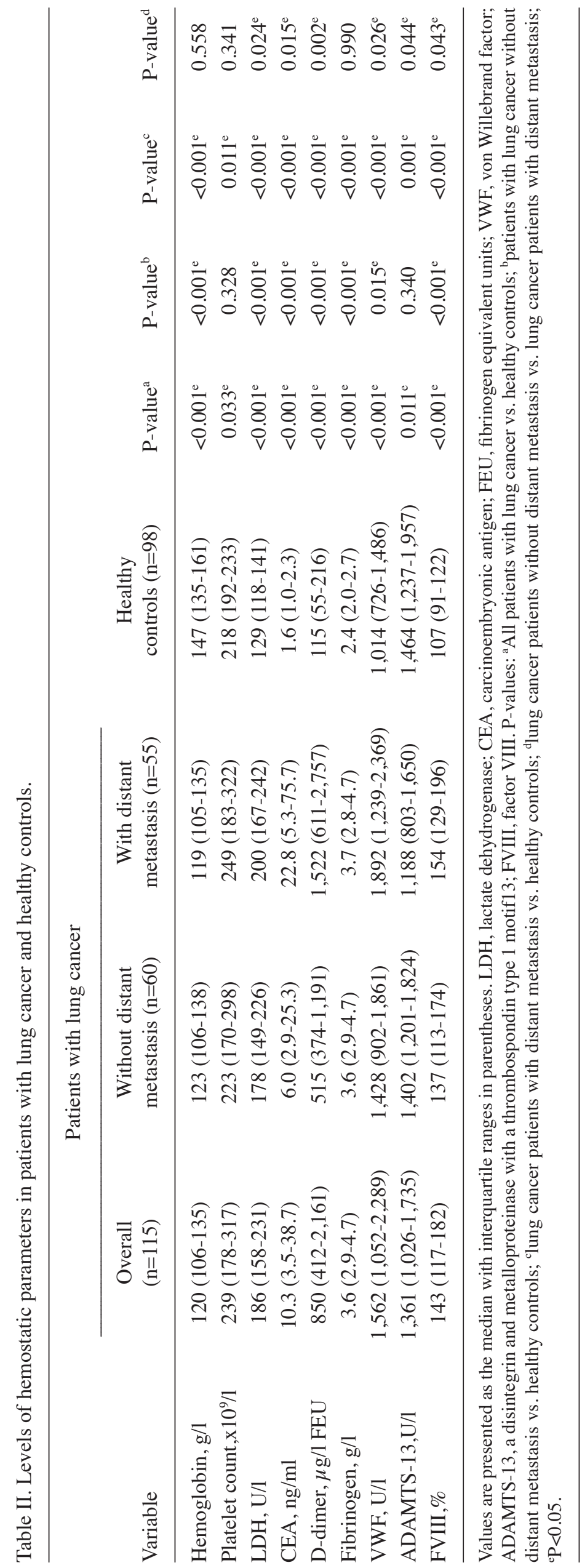


Table III. Comparison of hemostatic parameters in patients with lung cancer and healthy controls, according to the type $\mathrm{O}$ and non-O blood groups.

Patients with lung cancer

Healthy controls

\begin{tabular}{|c|c|c|c|c|c|c|}
\hline \multirow[b]{2}{*}{ Variables } & & & & \\
\hline & $O(n=29)$ & Non-O (n=86) & P-value & $O(n=34)$ & Non-O (n=64) & P-value \\
\hline Age, years & $56.9 \pm 10.0$ & $56.3 \pm 7.8$ & 0.892 & $55.7 \pm 7.6$ & $54.2 \pm 7.0$ & 0.931 \\
\hline Gender,male/female & $20 / 9$ & $56 / 30$ & 0.705 & $18 / 16$ & $40 / 24$ & 0.359 \\
\hline BMI, $\mathrm{kg} / \mathrm{m}^{2}$ & $22.1 \pm 2.4$ & $22.3 \pm 8.0$ & 0.802 & $22.4 \pm 3.0$ & $23.5 \pm 2.4$ & 0.751 \\
\hline SBP,mmHg & $126.4 \pm 9.8$ & $128.7 \pm 8.6$ & 0.291 & $125.3 \pm 10.8$ & $128.2 \pm 7.7$ & 0.337 \\
\hline $\mathrm{DBP}, \mathrm{mmHg}$ & $79.1 \pm 6.5$ & $80.7 \pm 6.8$ & 0.347 & $76.7 \pm 9.1$ & $79.5 \pm 6.4$ & 0.265 \\
\hline Smoking status, yes/no & $9 / 20$ & $23 / 63$ & 0.656 & $8 / 26$ & $12 / 52$ & 0.576 \\
\hline Distant metastasis,yes/no & $13 / 16$ & $42 / 44$ & 0.709 & - & - & - \\
\hline Hemoglobin, g/l & $128(115-138)$ & $118(104-134)$ & 0.140 & $143(134-158)$ & $149(136-161)$ & 0.359 \\
\hline Platelet count, $\times 10^{9} / 1$ & $227(175-294)$ & $241(179-322)$ & 0.604 & $212(186-229)$ & $220(200-242)$ & 0.083 \\
\hline LDH, U/1 & $180(158-238)$ & $189(157-230)$ & 0.879 & $125(116-142)$ & $131(120-141)$ & 0.745 \\
\hline $\mathrm{CEA}, \mathrm{ng} / \mathrm{ml}$ & $4.8(2.4-30.2)$ & $13.6(4.2-43.9)$ & 0.088 & $1.4(1.0-2.0)$ & $1.7(1.0-2.5)$ & 0.134 \\
\hline D-dimer, $\mu \mathrm{g} / 1 \mathrm{FEU}$ & $759(492-1,689)$ & $873(406-2,349)$ & 0.828 & $78(55-176)$ & $121(63-256)$ & 0.298 \\
\hline Fibrinogen, g/l & $3.6(2.8-4.7)$ & $4.1(3.1-4.9)$ & 0.440 & $2.1(2.0-2.5)$ & $2.6(2.1-2.8)$ & $0.004^{\mathrm{a}}$ \\
\hline VWF,U/1 & $1,190(892-1,865)$ & $1,676(1,099-2,383)$ & $0.022^{\mathrm{a}}$ & $865(583-1,291)$ & $1,117(772-1,649)$ & $0.006^{\mathrm{a}}$ \\
\hline ADAMTS-13, U/1 & $1,586(1,087-1,890)$ & $1,333(974-1,576)$ & 0.101 & $1,572(1,397-2,239)$ & $1,403(1,116-1,771)$ & $0.032^{\mathrm{a}}$ \\
\hline FVIII, \% & $130(113-161)$ & $145(124-194)$ & $0.014^{\mathrm{a}}$ & $98(81-113)$ & $112(100-124)$ & $0.003^{\mathrm{a}}$ \\
\hline
\end{tabular}

${ }^{\mathrm{a}} \mathrm{P}<0.05$. Values are presented as the mean \pm standard deviation or the median with interquartile ranges in parentheses. BMI, body mass index; DBP, diastolic blood pressure; SBP, systolic blood pressure; LDH, lactate dehydrogenase; CEA, carcinoembryonic antigen; FEU, fibrinogen equivalent units; VWF, von Willebrand factor; ADAMTS-13, a disintegrin and metalloproteinase with a thrombospondin type 1 motif13; FVIII, factor VIII.

type O. However, a significant decrease in ADAMTS-13 levels was observed only in the control group, not in the lung cancer group, among those with non-O type blood, as compared with those with type $\mathrm{O}$ blood $(\mathrm{P}<0.05$; Fig. $1 \mathrm{C})$. No significant differences were observed in platelet counts or serum levels of hemoglobin, LDH, CEA and D-dimer between the groups.

\section{Discussion}

Hemostatic abnormalities are present in $\sim 50 \%$ of patients with cancer and $>90 \%$ of those with metastatic disease (20). The results of the present study identified significantly increased plasma levels of hemoglobin, LDH, CEA, D-dimer, fibrinogen and VWF, and FVIII activity, in patients with lung cancer, regardless of the presence or absence of distant metastasis. These results confirmed an association between the activation of blood coagulation and fibrinolysis, and lung cancer, even though the underlying molecular mechanisms for this association remain incompletely understood. Additionally, significant differences in the plasma levels of LDH, CEA, D-dimer, VWF and ADAMTS-13, and FVIII activity were observed between patients with and without distant metastasis. These results are in agreement with those of previous studies, which demonstrated an increased tendency for the development of coagulative and fibrinolytic disorders in patients with metastatic diseases $(3,21)$. Furthermore, these results are similar to the observations of Oleksowicz et al (22), who identified increased VWF levels and ADAMTS-13 deficiencies in patients with metastatic tumors. Nevertheless, the results of the present study are in contrast with the results obtained by Martini et al (13), who identified a similar distribution of VWF between patients with lung cancer and control subjects. The ABO blood group is a major determinant of plasma VWF levels resulting in significantly decreased plasma levels in individuals with type $\mathrm{O}$ blood, as compared with those with non-O blood types (11). By contrast with the matched age and gender, and similar distribution of blood groups between cases and controls, as well as the strict inclusion and exclusion criteria of the subjects in the present study, the imbalance in the distribution of blood groups between cases and controls in the study by Martini et al (13) may explain the lack of differences in plasma VWF concentrations between the two groups.

Although the biological significance of hemostatic abnormalities in cancer remains unclear, evidence indicates that activation of the coagulative-fibrinolytic system by neoplastic cells may facilitate invasiveness and metastases (1). Indeed, elevated fibrinogen and D-dimer levels have been associated with unresponsiveness to treatment and unfavorable prognosis in patients with lung cancer (23). VWF is secreted from endothelial cells and readily degraded into small multimeric forms, which are more rapidly cleared from the circulation compared with the large multimeric forms. However, only the large multimeric forms of VWF are hemostatically active in the mediation of platelet adhesion to cancer cells (6). As ADAMTS-13 appears to be the most important protease that degrades VWF, ADAMTS-13 deficiency in patients with 


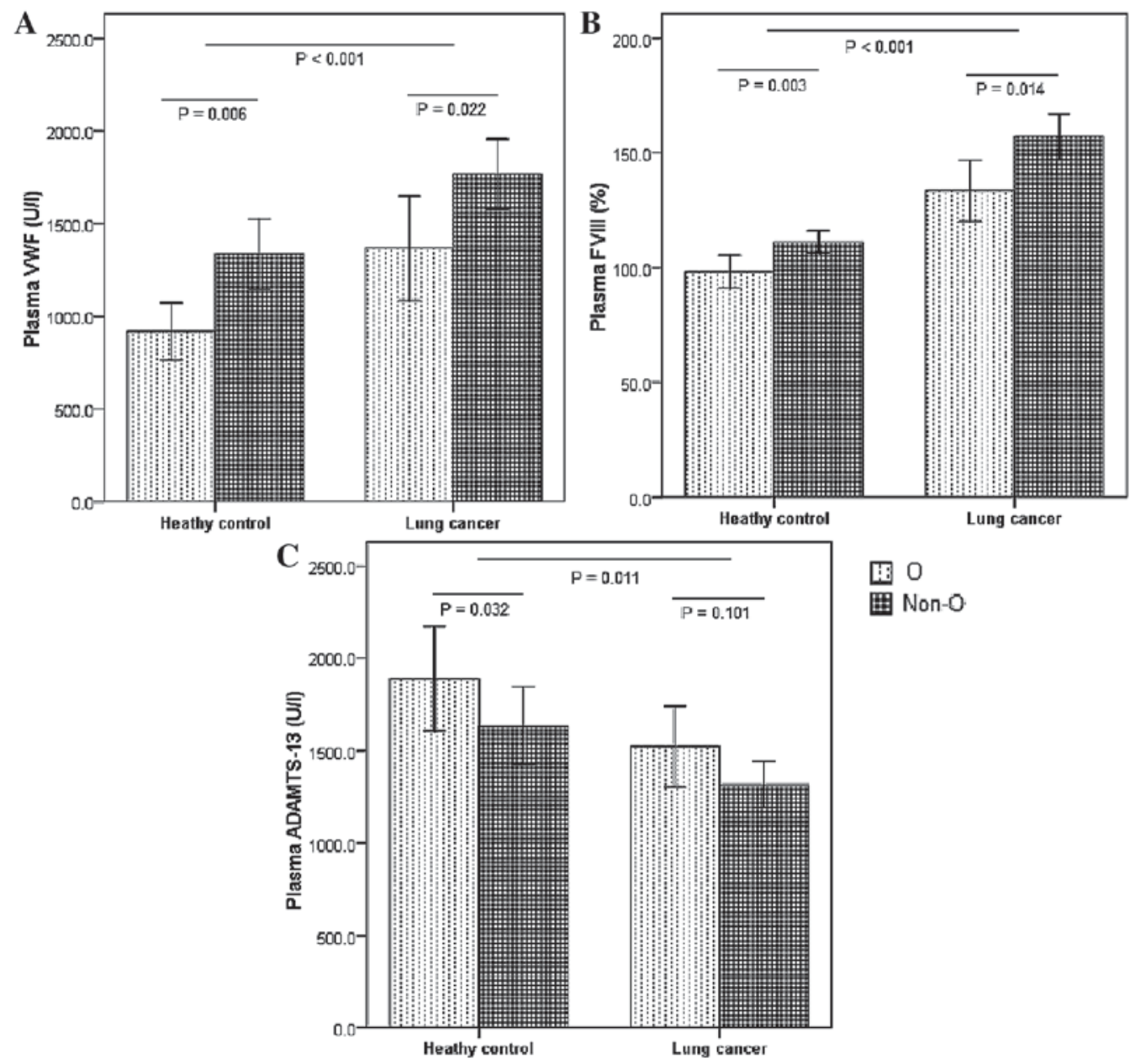

Figure 1. Comparison of plasma levels of (A) VWF, (B) FVIII and (C) ADAMTS-13 activity, according to O and non-O blood groups, in patients with lung cancer and healthy controls. VWF, von Willebrand factor; ADAMTS-13, a disintegrin and metalloproteinase with a thrombospondin type 1 motif 13; FVIII, factor VIII.

lung cancer may account for the appearance of large VWF multimers and increased VWF levels in circulating blood that may enhance tumor-induced platelet aggregation, resulting in thrombus formation and metastatic progression (24). Oleksowicz et al (22) demonstrated that platelet immunerelated glycoprotein Iba (GPIba) receptors may be expressed on the membrane of cancer cells with a particular affinity for large VWF multimers. The binding of GPIba receptors to large VWF multimers leads to the formation of platelet-cancer cell aggregates that allow the migration of cancer cells through vessel walls and the formation of novel sites of metastasis (25). However, the underlying molecular mechanism for ADAMTS-13 deficiency in cancer dissemination remains unclear. Therefore, stringent studies are required in order to establish a causal association between ADAMTS-13 deficiency and malignancy and/or metastasis.

Blood group antigens are chemical components on the erythrocyte membrane, but are also expressed on the surfaces of cancer cells. Alterations in blood group antigens may lead to alterations in the interactions between cells or cells and the extracellular matrix. These alterations are hypothesized to be important for the development of certain malignancies $(26,27)$. However, data concerning the role of ABO blood groups in lung cancer are limited and inconsistent (28-30). In the present study, no significant difference was observed between patients with lung cancer and healthy controls in terms of the distribution of ABO blood types, which is similar to the study by Oguz et al (28), but different from that of Urun et al (29), who identified an association between non-O blood types and an increased risk of lung cancer. This discrepancy may be due to differences in ethnicity, genetic variants, environmental factors and lifestyle factors among populations; therefore, inconsistent results may be obtained from varying genetic backgrounds and ethnicities (30). Linkage disequilibrium between $\mathrm{ABO}$ blood group genes and cancer-associated genes may influence cancer risk (29). Additionally, blood type may affect lifestyle, which was demonstrated to be associated with the incidence and mortality of lung cancer (31).

The ABO blood group has been associated with the development of venous thrombosis, coronary heart disease and ischemic stroke, since they are important determinants of VWF and FVIII plasma levels (11). It has been suggested that the carbohydrate moiety (ABH-bearing structures of VWF), similar to the blood group antigens $\mathrm{A}, \mathrm{B}$ and $\mathrm{H}(\mathrm{O})$, protects VWF from proteolytic degradation by ADAMTS-13 and may affect VWF function and, indirectly, FVIII levels (32). Bowen (33) demonstrated that proteolysis of VWF by ADAMTS-13 appears to be more rapid in patients with type O blood, compared with that in individuals with non-O blood types. The carbohydrate moiety also serves an important 
role in VWF polymerization and function, and affects the liver-mediated clearance of VWF (34). In addition, the ABO blood group locus on chromosome 9q34 is the most important genetic determinant of plasma levels of the VWF-FVIII complex (6). As VWF serves an important role as a carrier protein for FVIII, preventing its clearance from plasma, the majority of the effects of blood type on FVIII plasma levels are mediated by VWF (10). Additionally, several other factors, including fibrinogen, triacylglycerols, and genetic or acquired factors involved in the biosynthesis or clearance of VWF and FVIII, are also associated with increased plasma levels of FVIII (35). In the present study, FVIII activity and plasma levels of VWF were significantly increased in healthy controls with non-O blood types, compared with those with type $\mathrm{O}$ blood, which is in agreement with previously published data $(10,11)$. Similar results for FVIII activity and VWF levels were also observed in patients with lung cancer. As patients with distant metastasis have increased levels of VWF and FVIII, one would expect to observe an increased frequency of distant metastasis in patients with lung cancer with non-O type blood. However, no significant differences were observed in the frequencies of distant metastasis between the type $\mathrm{O}$ and non-O blood groups. Notably, decreased ADAMTS-13 levels were observed among those with non-O blood groups only in healthy controls, similar to a previous study that observed ADAMTS-13 levels were increased by $\sim 10 \%$ in individuals with type $\mathrm{O}$ blood, as compared with those with non-O type blood (36). Furthermore, the results of the present study indicated that plasma fibrinogen levels were significantly increased in healthy controls with non-O type blood, compared with in those with type $\mathrm{O}$ blood. However, no association of type $\mathrm{O}$ and non-O blood groups with fibrinogen and ADAMTS-13 levels was observed in patients with lung cancer. The small sample size of the present study may have led to insufficient statistical power. Another possible explanation for this result is that the state of hypercoagulability or acute inflammatory responses in patients with lung cancer may have surpassed the physiological influence determined by the ABO blood groups and subsequently masked the effect of blood type in this condition. These data confirm the presence of complicated alterations in the coagulative-fibrinolytic system in lung cancer, with a number of factors acting in concert.

The present study has a number of limitations that require addressing. First, the number of participants was small. However, to the best of our knowledge, the present study is the first to identify an association between ABO blood group and plasma levels of VWF and ADAMTS-13, and FVIII activity in patients with lung cancer. Secondly, ADAMTS-13 appears to be the most important protease that degrades VWF, whereas several proteases from granulocytes and platelets, which have also been demonstrated to degrade large VWF multimers in vitro, were not detected. Thirdly, measurement of the VWF antigen was insufficient to acquire specific information regarding circulating VWF molecular species, including the VWF propeptide and VWF multimers. Therefore, further studies are required to assess VWF propeptides, multimers and activity levels to reveal the imbalance between VWF secretion and ADAMTS-13 levels in patients with lung cancer.
The results of the present study demonstrated increased VWF levels and FVIII activity in patients with lung cancer with or without distant metastasis, whereas decreased ADAMTS-13 levels were observed in patients with disseminated tumors. Non-O blood groups constitute a risk factor for elevated plasma levels of FVIII and VWF activity in patients with lung cancer. It is reasonable to carefully monitor patients with lung cancer with non-O blood types to minimize the risk of thrombotic events. Furthermore, future studies are required to investigate potential associations between plasma VWF and ADAMTS-13 levels, and FVIII activity and outcomes of the patients with lung cancer with different ABO blood types.

\section{Acknowledgements}

The present study was financially supported by the Science and Technology Foundation of the Public Welfare Profession of Zhejiang Province (grant no. 2015C33198), the Department of Education Foundation of Zhejiang Province (grant no. Y201430628) and the Medical Science and Technology Project of Zhejiang Province (grant no. 2016KYA074).

\section{References}

1. Gabazza EC, Taguchi O, Yamakami T, Machishi M, Ibata H and Suzuki S: Evaluating prethrombotic state in lung cancer using molecular markers. Chest 103: 196-200, 1993.

2. Masago K, Fujita S, Mio T, Togashi Y, Kim YH, Hatachi Y, Fukuhara A, Irisa K, Sakamori Y and Mishima M: Clinical significance of the ratio between the alpha 2 plasmin inhibitor-plasmin complex and the thrombin-antithrombin complex in advanced non-small cell lung cancer. Med Oncol 28: 351-356, 2011.

3. Tas F, Kilic L, Serilmez M, Keskin S, Sen F and Duranyildiz D: Clinical and prognostic significance of coagulation assays in lung cancer. Respir Med 107: 451-457, 2013.

4. Terraube V, O'Donnell JS and Jenkins PV: Factor VIII and von Willebrand factor interaction: Biological, clinical and therapeutic importance. Haemophilia 16: 3-13, 2010.

5. Sporn LA, Marder VJ and Wagner DD: Inducible secretion of large, biologically potent von Willebrand factor multimers. Cell 46: 185-190, 1986.

6. Sadler JE: Biochemistry and genetics of von Willebrand factor. Annu Rev Biochem 67: 395-424, 1998.

7. Fujikawa K, Suzuki H, McMullen B and Chung D: Purification of human von Willebrand factor-cleaving protease and its identification as a new member of the metalloproteinase family. Blood 98: 1662-1666, 2001.

8. Moake JL: Thrombotic microangiopathies. N Engl J Med 347: 589-600, 2002.

9. Franchini M, Favaloro EJ, Targher G and Lippi G: ABO blood group, hypercoagulability, and cardiovascular and cancer risk. Crit Rev Clin Lab Sci 49: 137-149, 2012.

10. Jenkins PV and O'Donnell JS: ABO blood group determines plasma von Willebrand factor levels: A biologic function after all? Transfusion 46: 1836-1844, 2006.

11. Sousa NC, Anicchino-Bizzacchi JM, Locatelli MF, Castro V and Barjas-Castro ML: The relationship between ABO groups and subgroups, factor VIII and von Willebrand factor. Haematologica 92: 236-239, 2007.

12. Terraube V, Marx I and Denis CV: Role of von Willebrand factor in tumor metastasis. Thromb Res 120 (Suppl 2): S64-S70, 2007.

13. MartiniF,Ferroni P, Guadagni F, Basili S, Spila A,D'Alessandro R, Mineo D, Laudisi A, Portarena I, Mariotti S, et al: Plasma von Willebrand factor antigen levels in non-small cell lung cancer patients. Anticancer Res 25: 403-407, 2005.

14. Brambilla E, Travis WD, Colby TV, Corrin B and Shimosato Y: The new World Health Organization classification of lung tumours. Eur Respir J 18: 1059-1068, 2001.

15. Detterbeck FC, Boffa DJ and Tanoue LT: The new lung cancer staging system. Chest 136: 260-271, 2009. 
16. Bais R and Philcox M: Approved recommendation on IFCC methods for the measurement of catalytic concentration of enzymes. Part 8. IFCC method for lactate dehydrogenase (1-lactate: NAD+oxidoreductase, EC 1.1.1.27). International Federation of Clinical Chemistry (IFCC). Eur J Clin Chem Clin Biochem 32: 639-655, 1994.

17. Okuno T and Selenko V: Plasma fibrinogen determination by automated thrombin time. Am J Med Technol 38: 196-201, 1972.

18. Elf JL, Strandberg K and Svensson PJ: Performance of two relatively new quantitative D-dimer assays (Innovance D-dimer and AxSYM D-dimer) for the exclusion of deep vein thrombosis. Thromb Res 124: 701-705, 2009.

19. Barrowcliffe TW, Raut S, Sands D and Hubbard AR: Coagulation and chromogenic assays of factor VIII activity: General aspects, standardization, and recommendations. Semin Thromb Hemost 28: 247-256, 2002

20. Goad KE and Gralnick HR: Coagulation disorders in cancer. Hematol Oncol Clin North Am 10: 457-484, 1996.

21. Guadagni F, Ferroni P, Basili S, Facciolo F, Carlini S, Crecco M, Martini F, Spila A, D'Alessandro R, Aloe S, et al: Correlation between tumor necrosis factor-alpha and D-dimer levels in non-small cell lung cancer patients. Lung Cancer 44: 303-310, 2004.

22. Oleksowicz L, Bhagwati N and DeLeon-Fernandez M: Deficient activity of von Willebrand's factor-cleaving protease in patients with disseminated malignancies. Cancer Res 59: 2244-2250, 1999.

23. Unsal E, Atalay F, Atikcan S and Yilmaz A: Prognostic significance of hemostatic parameters in patients with lung cancer. Respir Med 98: 93-98, 2004

24. Sugimoto Y, Watanabe M, Oh-Hara T, Sato S, Isoe T and Tsuruo T: Suppression of experimental lung colonization of a metastatic variant of murine colon adenocarcinoma 26 by a monoclonal antibody 8F11 inhibiting tumor cell-induced platelet aggregation. Cancer Res 51: 921-925, 1991.

25. Oleksowicz L, Bhagwati N, Fernandez MD, Seno R and Etkind P: Prognostic significance of platelet immunorelated GPIb expression in breast cancer. Cancer J Sci Am 4: 247-253, 1998.

26. León-Atance P, Moreno-Mata N, González-Aragoneses F, Cañizares-Carretero MÁ,Poblet-Martínez E, Genovés-Crespo M, García-Jiménez MD, Honguero-Martínez AF, Rombolá CA Simón-Adiego CM, et al: Prognostic influence of loss of blood group A antigen expression in pathologic stage I non-small-cell lung cancer. Arch Bronconeumol 48: 49-54, 2012 (In English, Spanish).
27. Joh HK, Cho E and Choueiri TK: ABO blood group and risk of renal cell cancer. Cancer Epidemiol 36: 528-532, 2012.

28. Oguz A, Unal D, Tasdemir A, Karahan S, Aykas F, Mutlu H, Cihan YB and Kanbay M: Lack of any association between blood groups and lung cancer, independent of histology. Asian Pac J Cancer Prev 14: 453-456, 2013.

29. Urun Y, Utkan G, Cangir AK, Oksuzoglu OB, Ozdemir N, Oztuna DG, Kocaman G, Coşkun HS, Kaplan MA, Yuksel C, et al: Association of ABO blood group and risk of lung cancer in a multicenter study in Turkey. Asian Pac J Cancer Prev 14: 2801-2803, 2013

30. Amundadottir L, Kraft P, Stolzenberg-Solomon RZ, Fuchs CS, Petersen GM, Arslan AA, Bueno-de-Mesquita HB, Gross M, Helzlsouer K, Jacobs EJ, et al: Genome-wide association study identifies variants in the ABO locus associated with susceptibility to pancreatic cancer. Nat Genet 41: 986-990, 2009.

31. Suadicani P, Hein HO and Gyntelberg F: ABO phenotypes and inflammation-related predictors of lung cancer mortality: The Copenhagen Male Study-a 16-year follow-up. Eur Respir J 30: 13-20, 2007.

32. Klarmann D, Eggert C, Geisen C, Becker S, Seifried E, Klingebiel T and Kreuz W: Association of $\mathrm{ABO}(\mathrm{H})$ and I blood group system development with von Willebrand factor and factor VIII plasma levels in children and adolescents. Transfusion 50: 1571-1580, 2010.

33. Bowen DJ: An influence of ABO blood group on the rate of proteolysis of von Willebrand factor by ADAMTS13. J Thromb Haemost 1: 33-40, 2003

34. Wagner DD, Mayadas T and Marder VJ: Initial glycosylation and acidic $\mathrm{pH}$ in the Golgi apparatus are required for multimerization of von Willebrand factor. J Cell Biol 102: 1320-1324, 1986.

35. Choi Q, Kim JE, Kim SY, Han KS and Kim HK: Influence of ABO type on global coagulation assay results: Effect of coagulation factor VIII. Clin Chem Lab Med 53: 1425-1432, 2015.

36. Mannucci PM, Capoferri C and Canciani MT: Plasma levels of von Willebrand factor regulate ADAMTS-13, its major cleaving protease. Br J Haematol 126: 213-218, 2004. 